\title{
Comparative glycomic profiling in esophageal adenocarcinoma
}

\author{
Zane T. Hammoud, MD, ${ }^{\text {a }}$ Yehia Mechref, $\mathrm{PhD},{ }^{\mathrm{b}}$ Ahmed Hussein, $\mathrm{PhD},{ }^{\mathrm{b}}$ Slavka Bekesova, PhD, \\ Min Zhang, PhD, ${ }^{b}$ Kenneth A. Kesler, $\mathrm{MD},{ }^{\mathrm{c}}$ and Milos V. Novotny, $\mathrm{PhD}^{\mathrm{b}}$
}

\begin{abstract}
Objective: Aberrant glycosylation has been implicated in various types of cancers. Cancerous cells with altered glycosylation of their surface proteins shed such proteins into the circulating fluids. Glycomic profiling of such fluids shows the altered glycosylation. We performed glycomic profiling of serum from patients with no known disease, Barrett's without dysplasia, with high-grade dysplasia, and with esophageal adenocarcinoma in an attempt to delineate distinct differences in glycosylation among these groups.
\end{abstract}

Methods: Serum samples from patients with Barrett's metaplasia $(\mathrm{N}=5)$, high-grade dysplasia $(\mathrm{N}=11)$, and esophageal adenocarcinoma $(\mathrm{N}=50)$ were collected; samples from 18 healthy volunteers were used as control. Serum N-glycans were enzymatically released and then applied to both C18 Sep-Pak (Waters, Milford, MA) cartridges and activated charcoal cartridges. $\mathrm{N}$-glycans were permethylated and then spotted directly onto a matrixassisted laser desorption ionization plate. Mass spectra were acquired using the Applied Biosystems 4800 MALDI TOF/TOF Analyzer (Applied Biosystems Inc, Framingham, Mass). The obtained matrix-assisted laser desorption ionization-mass spectrometry data were processed using DataExplorer files (Applied Biosystems Inc) listing $\mathrm{m} / \mathrm{z}$ values and intensities.

Results: The intensities of 98 glycans were significantly different among the 3 groups; 26 of these corresponded to known glycan structures. Pairwise comparisons showed that 8 glycans were significantly different in all 3 pairwise comparisons.

Conclusion: We demonstrated that comparative glycomic profiling of esophageal adenocarcinoma reveals a subset of glycans that can be selected as candidate biomarkers. These markers can differentiate normal from high-grade dysplasia, normal from esophageal adenocarcinoma, and high-grade dysplasia from esophageal adenocarcinoma. Further validation will be necessary to determine the clinical utility of these glycan biomarkers. (J Thorac Cardiovasc Surg 2010;139:1216-23)

Esophageal adenocarcinomas are thought to develop from a premalignant lesion of the esophagus, referred to as Barrett's esophagus (BE). It is widely held that BE that eventually progresses to adenocarcinoma does so by a gradual progression at the cellular level from a normal squamous cell to the metaplastic columnar cell that is synonymous with BE. However, the molecular mechanisms by which such progression occurs and which patients with $\mathrm{BE}$ are at greatest risk for this progression have yet to be elucidated. A better understanding of the pathophysiology of Barrett's adenocarcinoma may help to identify those patients at increased risk for malignant transformation. The identification of novel biomarkers involved in the progression and ultimate malignant transformation has the potential to result in an earlier diagnosis and to improve the prognosis of patients with esophageal adenocarcinoma.

From the Cardiothoracic Surgery, Henry Ford Health System, ${ }^{a}$ Detroit, Mich; Indiana University, ${ }^{\mathrm{b}}$ Bloomington, Ind; and Indiana University School of Medicine, ${ }^{\mathrm{c}}$ Indianapolis, Ind.

Disclosures: None.

Received for publication April 30, 2009; revisions received Sept 26, 2009; accepted for publication Dec 11, 2009.

Address for reprints: Zane T. Hammoud, MD, Henry Ford Hospital, Cardiothoracic Surgery, K-14, 2799 W. Grand Blvd, Detroit, MI 48202 (E-mail: Zhammou1@ hfhs.org).

$0022-5223 / \$ 36.00$

Copyright (c) 2010 by The American Association for Thoracic Surgery doi: $10.1016 /$ j.jtcvs.2009.12.045
Aberrant glycosylation has been implicated in several types of cancer. ${ }^{1-3}$ Molecular changes in glycosylation also may be associated with the signaling pathways responsible for the malignant transformation of cells. ${ }^{4}$ Because cancer cells with altered glycosylation of their surface proteins eventually shed such proteins into the circulating fluids, glycomic profiling of such fluids may reveal the altered glycosylation. Glycomic profiling has been used to show glycan alterations of several cancers, including breast, ${ }^{5}$ ovarian, ${ }^{6}$ prostate $^{7}$ and hepatocellular ${ }^{8}$ carcinomas. We hypothesized that analysis of esophageal adenocarcinoma and its precursors would show altered glycosylation and identify candidate glycans that can differentiate or distinguish adenocarcinoma from its precursors. These altered glycans may then serve as potential biomarkers of esophageal adenocarcinoma and indicate proteins involved in disease progression. We performed comparative glycomic profiling of serum obtained from patients with no known disease, patients with $\mathrm{BE}$ without dysplasia, patients with $\mathrm{BE}$ and high-grade dysplasia (HGD), and patients with esophageal adenocarcinoma in an attempt to identify these altered glycans.

\section{MATERIALS AND METHODS \\ Collection of Serum Samples}

All work was conducted under an Indiana University Purdue University institutional review board-approved protocol. All subjects included in the 

Abbreviations and Acronyms
$\mathrm{BE}=$ Barrett's esophagus
HGD $=$ high-grade dysplasia
MALDI $=$ matrix-assisted laser desorption ionization
MS = mass spectrometry
ROC $=$ receiver operating characteristic

study provided informed consent according to institutional guidelines. Whole blood samples were collected from patients with histologically documented BE $(n=5), B E$ with $\operatorname{HGD}(n=11)$, and adenocarcinoma $(n=50)$. Blood samples from 18 healthy volunteers served as control. Each blood sample was allowed to clot for 45 minutes and then centrifuged at 2000 rpm for 10 minutes. The serum was aspirated, placed in a separate vial, and stored at $-80^{\circ} \mathrm{C}$ until use.

\section{Release of N-Glycans From Glycoproteins}

After thawing, a $10-\mu \mathrm{L}$ aliquot of serum was lyophilized and then resuspended in $100 \mu \mathrm{L}$ of $25 \mathrm{mmol} / \mathrm{L}$ ammonium bicarbonate. N-glycans were then enzymatically released using PNGase F according to our previously published procedure. ${ }^{9}$ A 5 mU aliquot of PNGase F was added to the reaction mixture and incubated overnight (18-22 hours) at $37^{\circ} \mathrm{C}$.

\section{Solid-Phase Extraction of Enzymatically Released N-Glycans}

The volume of enzymatically released glycans was adjusted to $1 \mathrm{~mL}$ by adding deionized water. Samples were then applied to both C18 Sep-Pak cartridges (Waters, Milford, Mass) and activated charcoal cartridges (Harvard Apparatus, Holliston, Mass). The reaction mixture was first applied to the $\mathrm{C} 18$ Sep-Pak cartridge that had been preconditioned with ethanol and deionized water according to the manufacturer's recommendation. The reaction mixture was circulated through the C18 Sep-Pak cartridge 5 times before washing with water. Peptides and O-linked glycopeptides were retained on the $\mathrm{C} 18$ Sep-Pak cartridge, and the released glycans were collected as eluents. Next, the C18 Sep-Pak cartridge was washed with $1 \mathrm{~mL}$ of deionized water. The combined eluents containing the released $\mathrm{N}$-glycans were then passed over activated charcoal microcolumns. The columns were preconditioned with $1 \mathrm{~mL}$ of acetonitrile and $1 \mathrm{~mL}$ of $0.1 \%$ trifluroacetic acid aqueous solution, as recommended by the manufacturer. After applying the sample, the microcolumn was washed with $1 \mathrm{~mL}$ of $0.1 \%$ trifluroacetic acid aqueous solution. The samples were then eluted with a $1-\mathrm{mL}$ aliquot of $50 \%$ acetonitrile aqueous solution containing $0.1 \%$ trifluroacetic acid. Finally, the purified glycans were evaporated to dryness using the vacuum CentriVap Concentrator (Labconco Corporation, Kansas City, Mo) before solid-phase permethylation.

\section{Solid-Phase Permethylation}

Permethylation of enzymatically released and solid-phase purified $\mathrm{N}$-glycans was accomplished using our recently published solid-phase permethylation technique. ${ }^{10,11}$ This permethylation procedure has been shown to minimize oxidative degradation and peeling reactions and to avoid the need for excessive clean-up. Samples were infused through the reactor at a slow flow rate of $2 \mu \mathrm{L} / \mathrm{min}$, as previously described. ${ }^{10,11}$ The reactor was then washed with $230 \mu \mathrm{L}$ acetonitrile (flow rate: $5 \mu \mathrm{L} / \mathrm{min}$ ). All eluents were combined, and the permethylated $\mathrm{N}$-glycans were finally extracted using $200 \mu \mathrm{L}$ chloroform and washed 3 times with $200 \mathrm{~mL}$ of water before drying.

\section{Matrix-Assisted Laser Desorption Ionization/Time of Flight Mass Spectrometry Instrumentation}

Permethylated glycans were resuspended in $2 \mathrm{~mL}$ of (50:50) methanol:water solution. A $0.5-\mu \mathrm{L}$ aliquot of the sample was then spotted directly on the matrix-assisted laser desorption ionization (MALDI) plate and mixed with the equal volume of 2,5 dihydroxybenzoic acid-matrix prepared by suspending $10 \mathrm{mg}$ of 2,5 dihydroxybenzoic acid in $1 \mathrm{~mL}$ of (50:50) water:methanol solution containing $1 \mathrm{mmol} / \mathrm{L}$ sodium acetate. The MALDI plate was then dried under vacuum to ensure uniform crystallization. Mass spectra were acquired using the Applied Biosystems 4800 MALDI TOF/TOF Analyzer (Applied Biosystems Inc, Framingham, Mass). This instrument is equipped with a neodymium-doped yttrium aluminium garnet laser with 355-nm wavelength. MALDI spectra were recorded solely in the positive-ion mode, because permethylation eliminates the negative charge normally associated with sialylated glycans.

\section{Data Evaluation}

MALDI mass spectrometry (MS) data were processed using DataExplorer 4.0 (Applied Biosystems Inc) to generate ASCII files listing mass/charge $(\mathrm{m} / \mathrm{z})$ values and intensities. An in-house developed software tool (PeakCalc 2.0) was then used to extract the intensities of N-glycans. Principal component analysis was performed using MarkerView (Applied Biosystems Inc), allowing the visualization of multivariate information. Supervised principal component analysis methods were used, with a prior knowledge of the sample groups as healthy versus diseased. MS data were weighted using the base-e logarithm of the peak intensities. The peak intensities were also scaled using the Pareto option, wherein each value is subtracted by the average and divided by the square root of the standard deviation. This option is suitable for MS data because it prevents intense peaks from completely dominating the principal component analysis process, thus allowing any peak with good signal-to-noise ratio to contribute. Receiver operating characteristic (ROC) curve analysis with AccuROC 2.5 software for Windows (Accumetric Corporation, Montreal, Quebec, Canada) was used to assess the sensitivity and selectivity of the potential diagnostic variables.

\section{RESULTS}

Table 1 shows the clinical stage of the 50 patients with esophageal adenocarcinoma; none of the patients received any therapy before serum collection. The intensity values of 421 features were collected from 18 control subjects, 5 patients with BE, 11 patients with HGD, and 50 patients with esophageal adenocarcinoma. Because the primary goal was to compare these groups in terms of the relative intensity values for each structure, and the number of BE samples was limited $(\mathrm{N}=5)$, the $\mathrm{BE}$ and HGD groups were combined. It is widely accepted that the total intensity of each individual varies; thus, relative intensity is used for comparison throughout the analysis described as follows.

Exploratory data analyses indicated that the relative intensity of the original data violated the normality and constant variance assumptions. After logarithm transformation of the relative intensity, normality was improved but still not achieved. As a result, a nonparametric method, the Kruskal-Wallis test, ${ }^{12}$ was used to test the equality of the medians. Because of the large number of tests, the Bonferroni method was used to correct for multiple comparisons with an overall type I error rate controlled at 0.05 . The statistical significance of each glycan was determined by comparing 
TABLE 1. Clinical stage of 50 patients with esophageal adenocarcinoma

\begin{tabular}{lc}
\hline Clinical stage & No. of specimens \\
\hline T1N0 & 6 \\
T2N0 & 5 \\
T3N0 & 6 \\
T2N1 & 1 \\
T3N1 & 14 \\
T4N1 & 1 \\
T2-3Nx & 2 \\
M1a & 7 \\
M1b & 7 \\
Unknown & 5 \\
\hline
\end{tabular}

the $P$ value from the Kruskal-Wallis test with $1.1876 \mathrm{e}-004$. If statistical significance was reached, comparisons of the glycan intensities between each pair of the 2 groups were performed using the Wilcoxon rank-sum test. ${ }^{13}$ All statistical analyses were carried out using R 2.5.0 and MATLAB version 7.4 (The MathWorks Inc, Natick, Mass).

Results from the Kruskal-Wallis test showed that the relative intensities of 98 features were significantly different among the 3 groups; of these, 26 glycans corresponded to known glycan structures. Further pairwise comparisons using the Wilcoxon rank-sum test showed that only 8 glycans with known structures were significantly different in all 3 pairwise comparisons, that is, comparisons between esoph- ageal adenocarcinoma and control, between esophageal adenocarcinoma and BE + HGD, and between control and BE + HGD. The other 18 glycans with known structures reached statistical significance in 2 of the 3 comparisons. On the basis of these results, a subset of glycans were selected as candidate biomarkers to differentiate all 3 groups or to differentiate 1 group from the other 2 groups.

Figure 1 demonstrates the plot of the scores of principal components 1 and 2 for all 4 groups. To a varying degree, the 4 sets of samples received distinguishable first principal component scores. Consequently, the 4 sets clustered in a manner representative of the glycomic profile differences for the 4 groups. However, samples from patients with BE were distinguishable from samples from patients with HGD only in the second principle component, suggesting limited differences between them. We also examined the changes in relative intensities of fucosylated (linkage of fucose to polypeptides) and sialylated (linkage of sialic acid to polypeptides) structures, because this is another means to monitor changes in glycosylation patterns. Figure 2 shows the results of this analysis. There was a statistically significant $(P<.005)$ decrease in total fucosylation of $\mathrm{N}$-glycans between controls and adenocarcinoma samples. A decrease was also observed for the BE and HGD samples, although this did not reach statistical significance. Unlike fucosylation, no significant change in sialylated $\mathrm{N}$-glycans was detected among the 4 groups.

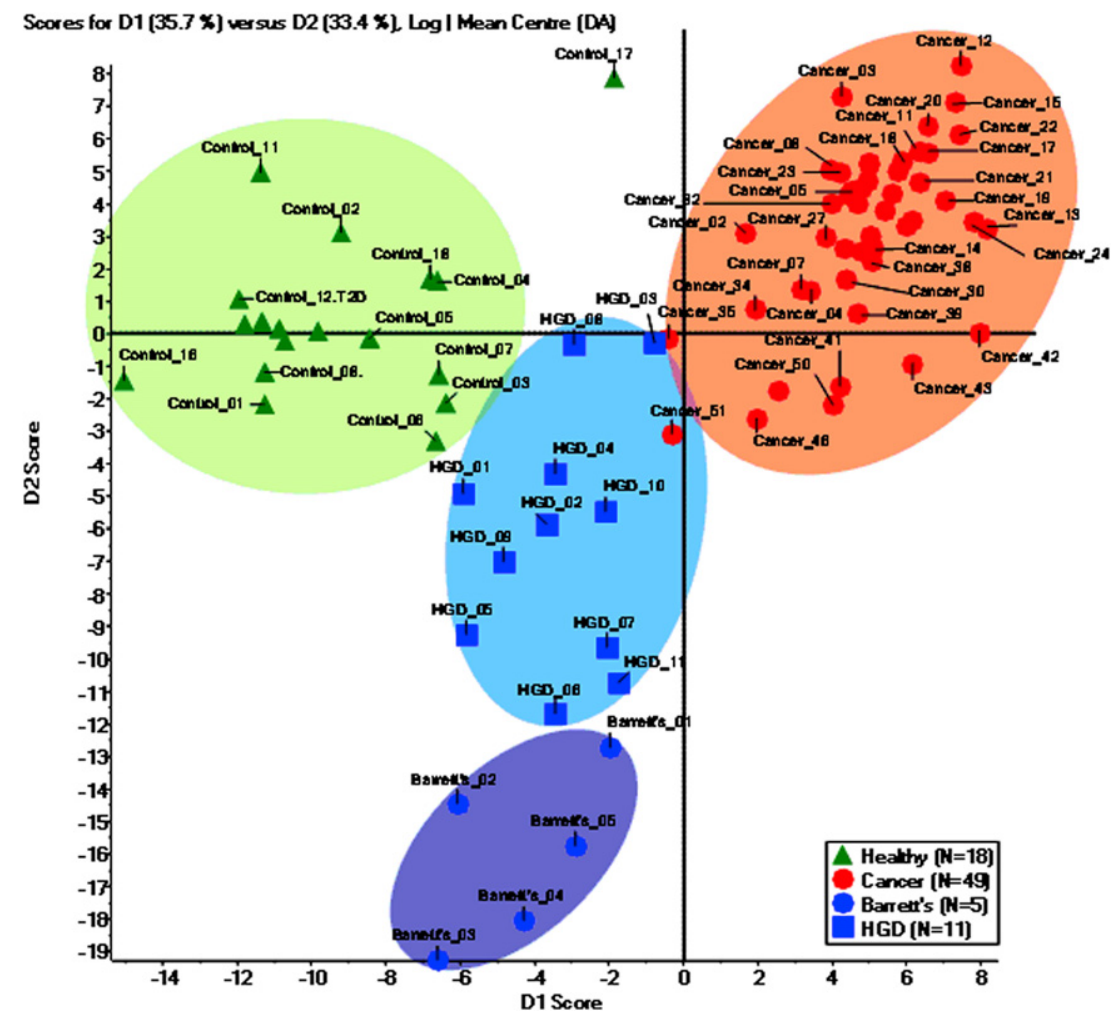

FIGURE 1. Plot of scores of principle components 1 and 2 for all 4 groups analyzed. $H G D$, High-grade dysplasia. 

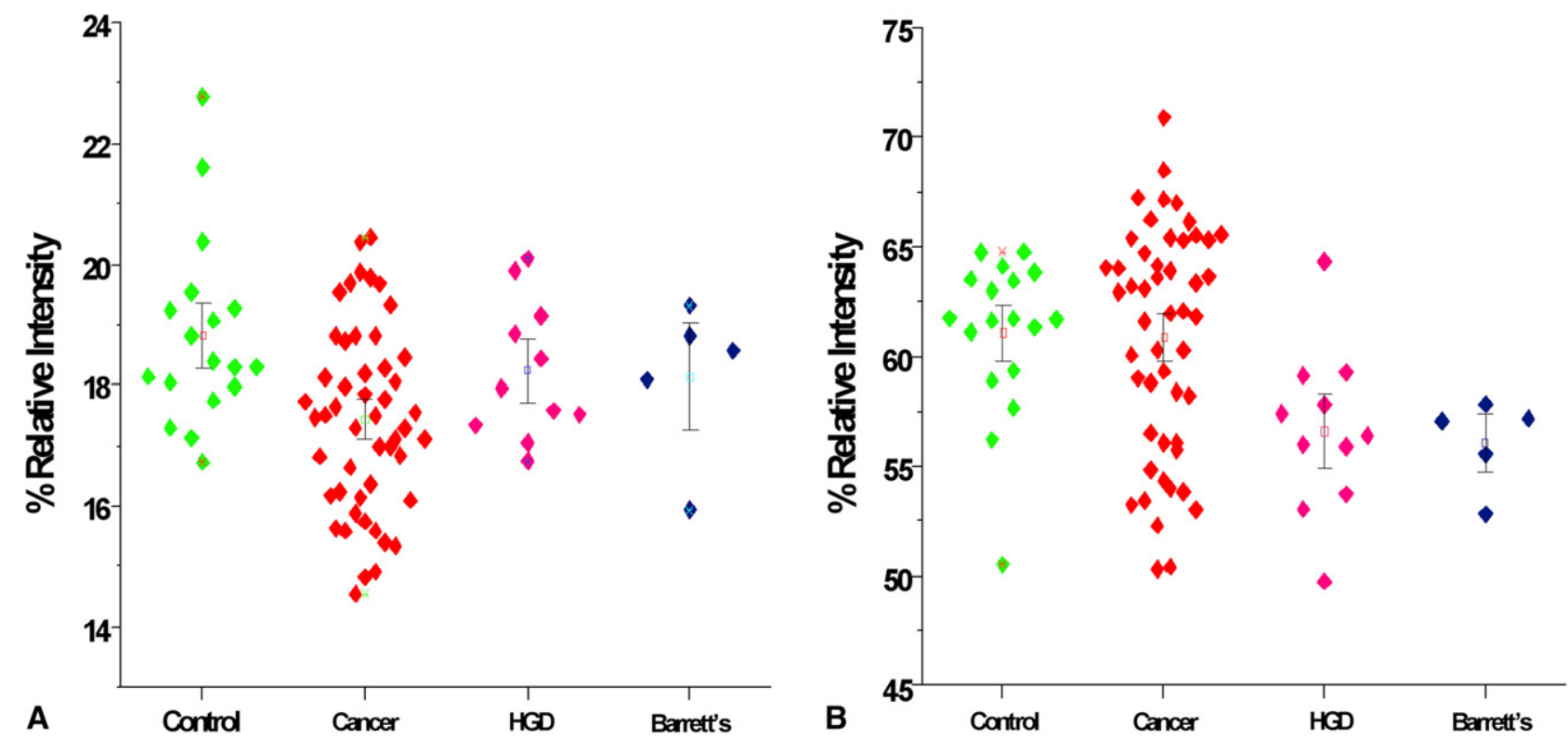

FIGURE 2. Results of analysis of relative intensities of fucosylated (A) and sialylated (B) glycans. HGD, High-grade dysplasia.

The changes in relative intensities associated with all known N-glycans observed in the MALDI-MS were further evaluated using 2 independent statistical approaches: Wilcoxon rank-sum and ROC curve analyses. The relative intensities of $16 \mathrm{~N}$-glycan structures were statistically different between control patients and patients with esophageal adenocarcinoma, as suggested by Wilcoxon rank-sum test $P$ values. These data are summarized in Figure 3. According to ROC analysis area under the curve values, 3 of these structures predict esophageal adenocarcinoma with high accuracy, whereas the other structures predict adenocarcinoma with moderate accuracy. The relative intensities of $14 \mathrm{~N}$-glycans were lower in patients with adenocarcinoma, whereas the relative intensities of 2 structures were higher. Dot-plots representing the relative intensities observed for some of the structures listed in Figure 3 are illustrated in Figure 4. The relative intensities of $16 \mathrm{~N}$-glycan structures were statistically different between controls and those with HGD by Wilcoxon rank-sum test $P$ values. According to ROC analysis area under the curve values, all of the structures predict HGD with only moderate accuracy. The relative intensities of $15 \mathrm{~N}$-glycan structures were statistically different between adenocarcinoma and HGD by Wilcoxon rank-sum test $P$ values. According to ROC area under the curve values, all of these structures predict HGD with only moderate accuracy. Figure 5 demonstrates the dotplot analysis of the individual clinical stage of the esophageal adenocarcinoma specimens.

\section{DISCUSSION}

Altered glycosylation has been demonstrated in a variety of cancers, including those of the breast, prostate, and liver. ${ }^{1-3}$
We have demonstrated altered glycosylation in esophageal adenocarcinoma and its known precursor lesions. It is well established that post-translational modification of proteins (eg, glycosylation) is a common means by which cell signaling is accomplished. With respect to glycoproteins, such modifications/alterations may provide insight into the mechanism(s) of altered glycosylation and indicate the structural changes of glycoproteins during carcinogenesis, thereby identifying potential biomarkers of disease development or progression. By using principle component analysis, we demonstrated a distinct clustering among the 4 sample groups analyzed, with a significant difference between controls and adenocarcinoma. We also noted an intermediate difference between controls and those with BE/HGD, suggesting that altered glycosylation may indicate disease progression. Such findings support the potential to use serum glycomic profiling to diagnose each disease state and monitor the progression of disease from BE/HGD to adenocarcinoma. Furthermore, altered glycosylation appears to differ in each stage of adenocarcinoma (Figure 5), although the numbers are too small to make any definitive statements in this regard.

Our group previously reported alterations in glycosylation in other types of cancers. ${ }^{7,8}$ The changes in the glycan structures we have reported thus far and in this article demonstrate limited but distinguishable overlap, thus suggesting they are cancer specific. However, thorough validation of such specificity is needed and currently being pursued. Another potentially interesting observation made in the current study is the finding of decreased fucosylation in esophageal adenocarcinoma specimens. This is not in agreement with the reported finding of increased fucosylation in other types of cancers, such as pancreas and colorectal cancers. ${ }^{14,15} \mathrm{We}$ also failed 


\begin{tabular}{|c|c|c|c|c|c|}
\hline Structure & Trend & $P$-value & ROC AUC & Sensitivity & specificity \\
\hline & Decrease & 2.60E-09 & $0.99 \pm 0.01$ & 100 & 92 \\
\hline & Decrease & $2.10 \mathrm{E}-07$ & $0.95 \pm 0.03$ & 100 & 90 \\
\hline & Decrease & $1.81 \mathrm{E}-08$ & $0.90 \pm 0.04$ & 83 & 86 \\
\hline & Decrease & 4.67E-05 & $0.88 \pm 0.05$ & 88 & 66 \\
\hline & Decrease & 4.13E-07 & $0.87 \pm 0.06$ & 77 & 82 \\
\hline & Increase & $6.70 \mathrm{E}-05$ & $0.86 \pm 0.06$ & 88 & 78 \\
\hline & Increase & $1.10 \mathrm{E}-05$ & $0.84 \pm 0.06$ & 66 & 82 \\
\hline & Decrease & $1.96 \mathrm{E}-08$ & $0.82 \pm 0.06$ & 83 & 64 \\
\hline & Decrease & 4.45E-07 & $0.79 \pm 0.08$ & 94 & 42 \\
\hline & Decrease & $1.17 \mathrm{E}-05$ & $0.79 \pm 0.08$ & 89 & 68 \\
\hline & Decrease & $8.43 \mathrm{E}-06$ & $0.77 \pm 0.08$ & 89 & 62 \\
\hline $\begin{array}{l}\Delta \\
\text { a }\{a\end{array}$ & Decrease & 2.22E-05 & $0.76 \pm 0.07$ & 83 & 86 \\
\hline & Decrease & $1.67 \mathrm{E}-07$ & $0.76 \pm 0.07$ & 89 & 86 \\
\hline Or \{ & Decrease & 2.49E-06 & $0.75 \pm 0.07$ & 89 & 58 \\
\hline 舍 & Decrease & 0.0002 & $0.74 \pm 0.08$ & 78 & 78 \\
\hline$\checkmark\left\{\begin{array}{l}0 \\
0,0\end{array}\right.$ & Decrease & 0.007 & $0.74 \pm 0.08$ & 83 & 66 \\
\hline
\end{tabular}

FIGURE 3. Area under the curve values from receiver operating characteristic analysis, $P$ values of Wilcoxon rank-sum test, and test sensitivity and specificity for $\mathrm{N}$-glycans derived from human blood serum of control patients and patients with esophageal adenocarcinoma. Green = mannose; yellow $=$ galactose blue $=\mathrm{N}$-acetylglucosamine; red $=$ fucose; purple $=\mathrm{N}$-acetylneuraminic acid. The first 3 rows are the $\mathrm{N}$-glycans that received highly accurate ROC analysis AUC values (AUC $>0.8$ ) and low $P$ values in the Wilcoxon rank-sum test, whereas others are N-glycans that received moderately accurate ROC analysis AUC values $(0.7<\mathrm{AUC}<0.8)$ and low $P$ values in the Wilcoxon rank-sum test. $A U C$, Area under the curve; $R O C$, receiver operating characteristic.

to observe any alteration in sialylation, although other similar studies have reported altered sialylation in various cancer types. ${ }^{16,17}$ Such observations suggest that these findings may be unique to esophageal adenocarcinoma and that glycomic profiling is disease specific, thus supporting its potential use as a diagnostic tool.

\section{Study Limitations}

There are several limitations to our work. The number of samples analyzed is small, and further work will be needed to validate the findings. Ideally, the results obtained in the current study can serve as a test set, and "blinded" analysis of further additional specimens will serve to validate our 


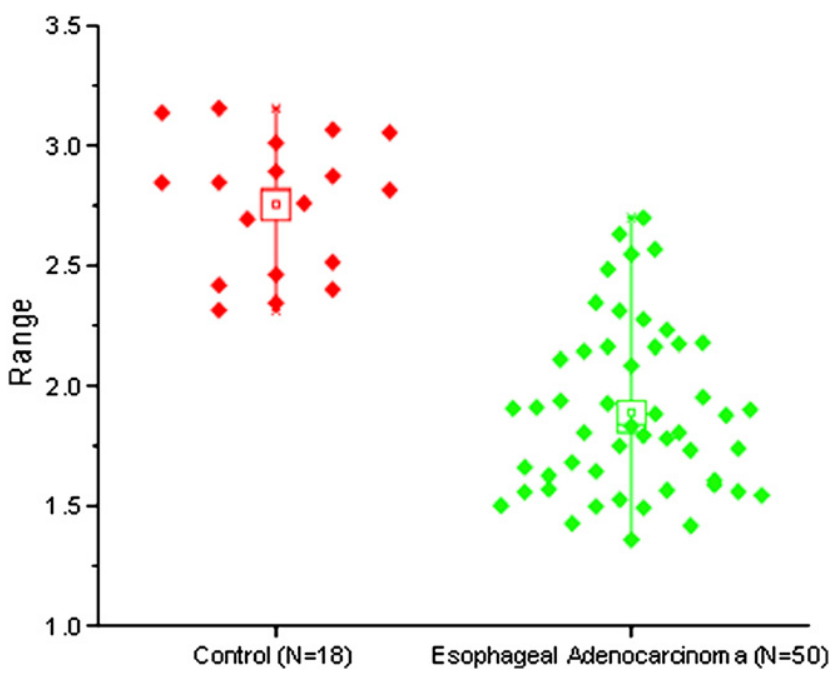

FIGURE 4. Dot-plot of the first 4 structures listed in Figure 3 (sensitivity 100 , specificity 96).

results. However, we consider this study exploratory in nature and are currently analyzing additional specimens in an attempt to validate our findings. Other disease states, such as esophagitis and other types of tumors, must also be analyzed and compared. Furthermore, only a prospective, blinded analysis will determine the ultimate validity and potential clinical utility of glycomic profiling. Another potential limitation is the ability to reproduce the results. The repeatability of MS for glycomic analysis was addressed in a pilot study conducted by the Human Proteome Organisation Human Disease Glycomics/Proteome Initiative and included 20 different laboratories, including ours. The study involved comparing the MS analysis of $\mathrm{N}$-linked glycans of standard samples acquired in 20 laboratories $^{18}$ and concluded that in general, MALDI/time of flight MS of permethylated oligosaccharide mixtures performed in 6 laboratories yielded good quantitation. The study also revealed the high repeatability and reproducibility of MS analysis of permethylated glycans. There are also confounding variables that have the potential to alter any analysis of blood serum, including comorbid conditions, diet, age, and gender. Although it is difficult, if not impossible, to control for all such factors, the patterns observed between individuals in each of the 4 sample groups were remarkably consistent, suggesting that the findings were not highly affected by such factors. We also obtained all samples from

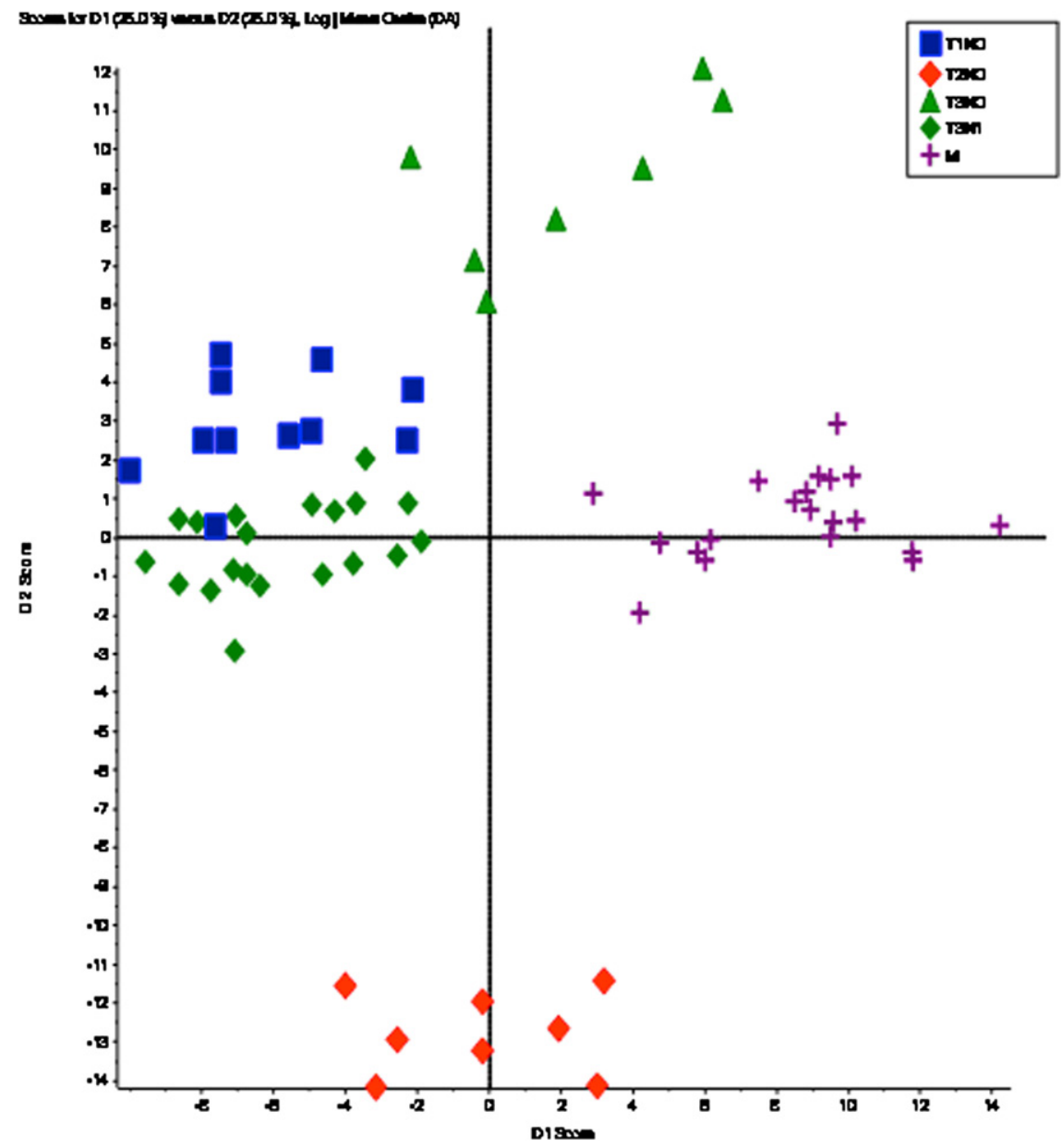

FIGURE 5. Dot-plot analysis of various clinical stages of esophageal adenocarcinoma. 
patients in the fasting state in an attempt to control for dietary consumption. In addition, the control group should also be closely matched to the disease group with regard to age, gender, and so forth. However, we wanted controls to be free of any possible disease that may confound our results. Given the typical age and comorbid conditions of patients with esophageal adenocarcinoma, it was exceedingly difficult to find controls in a similar age group without any comorbid conditions. We also do not have information regarding the proteins that underwent the detected glycomic changes. This endeavor will be undertaken once our data have been validated. It should be noted, however, that the mere identification of a glycomic "pattern" or "signature" may nonetheless prove clinically useful even in the absence of the identification of the specific protein(s).

\section{CONCLUSIONS}

We demonstrated the potential of MS-based serum glycomic profiling in the diagnosis of esophageal adenocarcinoma and its precursor lesions. The data provide some insight into the mechanisms of altered glycosylation and may thus indicate structural changes in glycoproteins. Such methodologies may form the basis for future clinical applications, such as disease screening or monitoring through the detection of glycan-specific biomarkers. Further validation and future prospective studies are needed to confirm the utility of glycomic profiling.

\section{References}

1. Baldus SE, Wienand JR, Werner JP, Landsberg S, Drebber U, Hanisch FG, et al. Expression of MUC1, MUC2 and oligosaccharide epitopes in breast cancer: prognostic significance of a sialylated MUC1 epitope. Int J Oncol. 2005;27:1289-97.

2. Dwek MV, Lacey HA, Leathem AJ. Breast cancer progression is associated with a reduction in the diversity of sialylated and neutral oligosaccharides. Clin Chim Acta. 1998;271:191-202.

3. Handerson T, Camp R, Harigopal M, Rimm D, Pawelek J. Beta 1,6-branched oligosaccharides are increased in lymph node metastases and predict poor outcome in breast carcinoma. J Clin Cancer Res. 2005;11:2969-73.

4. Alper J. Glycobiology: turning sweet on cancer. Science. 2003;301:159-60.

5. Kyselova Z, Mechref Y, Kang P, Goetz JA, Dobrolecki LE, Sledge G, et al. Breast cancer diagnosis and prognosis through quantitative measurements of serum glycan profiles. Clin Chem. 2008;54:1166-75.

6. An HJ, Miyamoto S, Lancaster KS, Kirmiz C, Li B, Lam KS, et al. Profiling of glycans in serum for the discovery of potential biomarkers for ovarian cancer. J Proteome Res. 2006;5:1626-35.

7. Kyselova Z, Mechref Y, Al Bataineh MM, Dobrolecki LE, Hickey RJ, Vinson J, et al. Alterations in serum glycome due to metastatic prostate cancer. J Proteome Res. 2007;6:1822-32.

8. Goldman R, Ressom H, Varghese R, Goldman L, Bascug G, Loffredo C, et al. Detection of hepatocellular carcinoma using glycomic analysis. Clin Cancer Res. 2009;15:1808-13.

9. Mechref Y, Novotny MV. Mass spectrometric mapping and sequencing of $\mathrm{N}$-linked oligosaccharides derived from submicrogram amounts of glycoproteins. Anal Chem. 1998;70:455-63.

10. Kang P, Mechref Y, Klouckova I, Novotny MV. Solid-phase permethylation of glycans for mass spectrometric analysis. Rapid Commun Mass Spectrom. 2005; 19:3421-8.

11. Kang P, Mechref Y, Novotny MV. High-throughput solid-phase spin-column permethylation for MALDI-TOF-MS analysis. Rapid Commun Mass Spectrom. 2008;22:721-34.

12. Kruskal WH, Wallis WAJ. Errata to use of ranks in one-criterion variance analysis. J Am Stat Assoc. 1952;47:583-621.
13. Wilcoxon F. Individual comparisons by ranking methods. Biometrics. 1945;1: 80-3.

14. Mas E, Pasqualini E, Caillol N, El Battari A, Crotte C, Lombardo D, et al. Fucosyltransferase activities in human pancreatic tissue: comparative study between cancer tissues and established tumoral cell lines. Glycobiology. 1998;8:605-13.

15. Izawa M, Kumamoto K, Mitsuoka C, Kanamori C, Kanamori A, Ohmori K, et al. Expression of sialyl 6-sulfo Lewis $\mathrm{X}$ is inversely correlated with conventional sialyl Lewis X expression in human colorectal cancer. Cancer Res. 2000;60: 1410-6.

16. Chandrasekaran EV, Xue J, Xia J, Chawda R, Piskorz C, Locke RD, et al. Analysis of the specificity of sialyltransferases toward mucin core 2, globo, and related structures. identification of the sialylation sequence and the effects of sulfate, fucose, methyl, and fluoro substituents of the carbohydrate chain in the biosynthesis of selectin and siglec ligands, and novel sialylation by cloned $\alpha 2,3(\mathrm{O})$ sialyltransferase. Biochemistry. 2005;44:15619-35.

17. Peracaula R, Tabares G, Royle L, Harvey DJ, Dwek RA, Rudd PM, et al. Altered glycosylation pattern allows the distinction between prostate-specific antigen (PSA) from normal and tumor origins. Glycobiology. 2003;13:457-70.

18. Wada Y, Azadi P, Costello CE, Dell A, Dwek RA, Geyer H, et al. Comparison of the methods for profiling glycoprotein glycans-HUPO Human Disease Glycomics/Proteome Initiative Multi-Institutional Study. Glycobiology. 2007;17: 411-22.

\section{Discussion}

Dr A. Pennathur (Pittsburgh, Pa). Dr Hammoud, that was an excellent presentation and a very interesting study. In your study you examined the serum samples of 5 patients with BE, 11 patients with HGD, and 50 patients with esophageal cancer, the majority of whom had locally advanced or metastatic disease. You identified 26 known glycan structures that were significantly different, and among these, 8 were different in all pairwise comparisons. You concluded that a subset of glycans can be selected as candidate biomarkers for the differentiation of controls, BE, and esophageal cancer and that further validation will be necessary. This is an interesting study, and I agree that further validation will definitely be necessary. One of the difficulties with serum proteomics or profiling is to standardize the conditions at the time of collection of the sample. Were these samples obtained in the office or the operating room, was the patient awake or under anesthesia, and were these samples handled similarly across the spectrum of all these categories?

Dr Hammoud. They were collected in the clinic. We made sure that patients were in a fasting state when they came to see us, and they were all done by the same person in terms of isolating the serum so we could control the protocol.

Dr Pennathur. Excellent. Were these samples from patients with HGD obtained in patients who had undergone esophagectomy? As you well know, several surgical series have demonstrated occult cancer in approximately $40 \%$ of patients with HGD who had undergone esophagectomy. The serum sample from a small sample size of 11 patients with HGD determined purely by endoscopy may confound your results.

Dr Hammoud. That is absolutely true. There were 6 patients in that cohort who underwent surgery and at surgery were documented and confirmed to have only HGD. The other 5 were endoscopic procedures.

Dr Pennathur. Are you planning any longitudinal follow-up in your patients with $\mathrm{BE}$ to determine changes in the profile, and do we know which proteins are glycosylated?

Dr Hammoud. No, we are not longitudinally following them, and we have not yet gotten to the point where we're looking at the proteins involved. 
Dr D. Harpole (Durham, NC). This is fascinating. As a next step, obviously you could perform MALDI in another set of patients and see if you come up with the same profile, and you may or may not. What would be your step in making this a more userfriendly test? In other words, are you going to try and identify the proteins through MS/MS and develop a serum enzyme-linked immunosorbent assay test or something for the glycosylated proteins you have discovered, or do you think this is just exploratory and you actually want to have a larger patient population and refine your dataset before you develop a test?

Dr Hammoud. The latter. We definitely need more sample sets. As you know, even in Pittsburgh and large centers, they only see approximately 150 of these cases per year. We see approximately 50 to 60 cases per year. So I need several years to collect the specimens and have a large enough pool to refine the data. My ultimate goal is not really the proteins. I think that's kind of a Holy Grail, kind of like looking for the cancer suppressor gene that's responsible for something. My ultimate goal is to generate MS profiles, and if we can identify and correlate a profile that says HGD in a patient is going to progress to adenocarcinoma, I consider that a clinically relevant thing to do.

Dr Harpole. A MALDI fingerprint, in other words.

Dr Hammoud. Correct. 